\title{
STRUCTURAL BASIS OF INTERFACE ENGINEERING
}

\author{
W.A. ŚWIATTICKI \\ Faculty of Materials Science \& Engineering, Warsaw University of Technology \\ Narbutta 85, 02-524 Warszawa, Poland
}

The paper analyses in a systematic manner these structural factors of interfaces in relation to their properties that constitute the background of "interface engineering". It is shown that the specific properties of interfaces are related to their structure. A brief description of recent structural models of interfaces is therefore presented. A new concept of interface microstructure composed of different kinds of interfacial defects is introduced and their role in determining the interface properties is emphasised. It is shown that interfaces control the processes taking place in the material under external solicitations or during the microstructure formation, mainly through their interaction with other crystal defects. Various examples illustrating the possibility of controlling and improving the material properties by appropriate changes of interface structures are presented in the case of metals, alloys, intermetallics and ceramics.

PACS numbers: $61.72 . \mathrm{Mm}$

\section{Introduction}

One of the basic ideas of materials science is that the properties of crystalline materials depend on their microstructure. The microstructure describes a spatial distribution of phases and different lattice defects (called microstructure elements) occurring inside the phases and between them [1]. The behaviour of the materials is therefore controlled by complex processes in which the elements of microstructure participate. The common microstructure elements of polycrystals are intercrystalline interfaces, which are the quasi-planar regions joining together neighbouring grains. An interface may join two different phases or two misoriented grains of the same phase which are called interphase boundary (IB) and grain boundary (GB), respectively. A polycrystal may be considered as composed of two different components: grains and interfaces, which form a three-dimensional network. It follows that the properties of polycrystalline materials result from both components and in many cases are mostly determined by the interfaces. It was observed that interfaces strongly influence such material properties as mechanical $[2,3]$, corrosive [4], electronic [5], superconducting [6-8] and others. The interfaces may deteriorate or 
improve the material properties [9]. Finally they control the kinetics of microstructural processes and therefore influence the fabrication of materials (e.g. sintering) and microstructure formation (grain growth, recrystallization, phase transformation).

For a long time it was impossible to control the influence of interfaces on material properties due to insufficient theoretical and technological knowledge. Recent progress in the theoretical description and modelling of interface structures and in experimental methods of investigating their chemical and physical characteristics, has permitted selection of these structural factors which control interfacial properties. The appropriate uses of these factors enable one to design the interface structure and intrinsic properties and therefore, to control the macroscopic properties of polycrystalline materials. This general idea was appeared many years ago, independently in different laboratories, among others in Grabski's group, and was developed about 10 years ago in the more rigorous scientific approach called "interface engineering" $[2,3]$. This approach is based on: (a) relationships between interface structure and its properties, (b) relationships between the collective behaviour of interfaces with different structures and macroscopic properties of material.

The paper analyses systematically these structural factors of interfaces in relation to their properties that constitute the background of "interface engineering". The essential problem of interface engineering deals with the nature of interfacial properties and this will be presented first.

\section{Interfacial properties}

\subsection{Intrinsic interfacial properties}

A property of an interface can generally be defined by the kind, magnitude and/or intensity of their response upon external solicitations (mechanical, thermal electrical, etc.). To measure the interfacial property it is therefore necessary to determine the functions and/or parameters that relate their response to the solicitations. It is generally observed that the response of an interface is different from that of the crystal; interfaces display a higher diffusivity [10], they are more susceptible to the corrosive environment [11] and can be more brittle than crystals [9]. Hence we can define such properties as interfacial resistivity, strength, viscosity...

\subsection{Interface controlled processes and reactions}

As the interface is not a separate object but an integral element of the material microstructure, the macroscopic external solicitations are generally transformed to internal solicitations occurring at microscopic scale. Many of these involve or result directly from the action of other crystal defects, such as point defects or dislocations. This leads to the complex reactions between the elements of crystal microstructure and the interfaces. For instance, the external mechanical stresses give rise to the internal local stress concentrations created by dislocations' pile-ups at the interfaces. When the stress increases the response of interface may 
consist of an interfacial fracture [2] or complex dislocation reactions such as: slip transmission across the interface, dislocation's generation, or dissociation and accommodation of dislocations into the interface $[3,12]$. It follows that the interfaces can act as sources and sinks of other crystal defects and in this way they may influence the material properties indirectly.

Exact knowledge of interface interactions with other crystal defects and processes occurring at microscopic level is therefore essential for understanding the role of interfaces in the macroscopic properties of a material. To obtain this piece of information it was necessary to develop specific methods for measuring the interfacial properties.

\subsection{Measurement of interfacial properties}

One of the most difficult problems related to interfacial property measurements deal with the scale length of this microstructural element and their environment. An interface is a planar defect of an area, usually of a few thousands square micrometers and the thickness of a few atomic distances. Thus, to determine intrinsic properties of individual boundaries, some special microscopic methods are used. These are, for example, the in situ methods consisting of annealing or straining the interfaces directly into the electron microscope for measurements of interfacial diffusivity [13-15] and mechanical properties [16], respectively. The in situ measurements of the electron beam induced current (EBIC) [17] and local resistivity [18] can be applied to determine the electrical properties of individual interfaces. Moreover, an interface cannot act independently of its environment, which makes it impossible to measure interfacial properties separately from the matrix. For example, there are not any direct methods of determining the absolute values of free interfacial energy. The commonly used indirect methods based on interfacial faceting [19], GB dissociation [5,20] or rotating crystallite experiments $[21,22]$, lead to the qualitative estimation of interfacial energy only. More quantitative, direct methods consisting of measurements of dihedral angles at triple junctions, or at free surfaces during thermal grooving experiments give rise to the relative values of interfacial energy only [23]. Many other interfacial properties are difficult to obtain directly.

\subsection{Interfacial energy}

The free interfacial energy $\gamma_{b}$ is the most fundamental interfacial property and can be expressed as [24]

$$
\gamma_{b}=E_{b}-T S_{b}
$$

where $E_{b}$ is the interfacial energy and $S_{b}$ is the interfacial entropy term.

The interfacial free energy describes the excess energy of the system containing an interface compared to the perfect lattice. The excess comes from the disturbed electronic structure at the interface, unsaturated bonds and/or from elastic stresses created by local lattice distortions [25]. The $\gamma_{b}$ values are mainly determined by experimental measures while $E_{b}$ is rather obtained by calculations. The entropy term in Eq. (1) remains the most difficult factor to quantify. 
It can be shown that the interfacial energy is a basic quantity for others interfacial properties and processes such as interfacial fracture, diffusion or mobility [23-25].

For example one of the most relevant properties for mechanical behaviour of materials is the work of fracture equal to the adhesive energy $W_{\mathbf{A B}}$ between two crystals $A$ and $B$ which can be expressed by the following equation [26]:

$$
W_{\mathrm{AB}}=\gamma_{\mathrm{A}}+\gamma_{\mathrm{B}}-\gamma_{b},
$$

where $\gamma_{A}$ and $\gamma_{B}$ are surface energies of crystal $A$ and $B$, respectively.

This equation indicates that the resistance to interfacial fracture increases when the interfacial energy $\gamma_{b}$ decreases.

From the kinetic point of view, the important property directly related to the interface core is the interfacial diffusivity $D_{b}$ which can be expressed by

$$
D_{b}=D_{b}^{0} \exp \left(-Q_{b} / R T\right)
$$

where $D_{b}^{0}$ and $Q_{b}$ are the preexponential factor and the activation energy of interfacial diffusion, respectively, $R$ is the gas constant and $T$ - absolute temperature.

Interfacial diffusivity is related to the interfacial energy by the following relation [27]:

$$
\gamma_{b}=\left[R T \ln \left(D_{b} / D_{1}\right)\right] / 2 a^{2} N_{0}=\left[R T \ln \left(D_{b}^{0} / D_{1}^{0}\right)+\left(Q_{1}-Q_{b}\right)\right] / 2 a^{2} N_{0},
$$

where $D_{1}$ is lattice diffusivity, $D_{1}^{0}$ and $Q_{1}$ are preexponential factor and activation energy of lattice diffusion, respectively, $a-$ a mean interatomic distance at the interface and $N_{0}$ - Avogadro number.

Boundary diffusivity is, in general, greater than that of the matrix and for high energy boundaries the difference can reach three orders of magnitude [28]. Thus the interfacial diffusivity directly controls many high temperature processes occurring at the boundaries during microstructure transformations (recrystallization, grain growth, interfacial precipitation) or during external solicitations (creep deformation) [23].

\subsection{Variability of interfacial properties}

It is worth noticing that the type and magnitude of interfacial response upon external forces differ strongly between different interfaces. The effect that particular interfaces behave differently was observed during measurements of interfacial energy [23-25,29], fracture $[2,9,26]$, sliding [30], resistivity $[7,8,18]$, and corrosion $[4,11]$. The dependence of interfacial energy on the misorientation angle for a chosen rotation axis displays cusps of various depths for certain angular values [23, 31] (Fig. 1). The similar, non-monotone dependence of misorientation angle has also been observed for other interfacial properties such as intergranular GB embrittlement [32], sliding [23,30] and corrosion [4, 11]. Furthermore, different interfaces are more or less effective sources, barriers or sinks for lattice defects. It has been shown that the interfacial properties depend also strongly on the orientation of interfacial plane for the same misorientation. Moreover, some interfacial properties as for example interfacial diffusion or GB sliding are highly anisotropic $[23,30]$. 


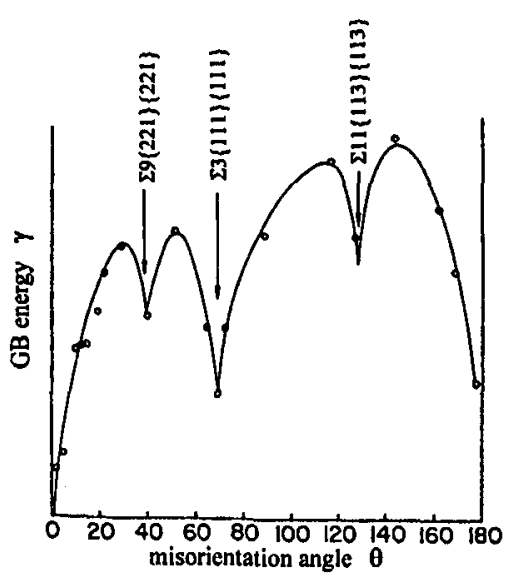

Fig. 1. Grain boundary energy $\gamma$ versus tilt angle $\theta$ for $\langle 110\rangle$ tilt boundaries in NiO (by courtesy of M. Déchamps).

A question arises: what is the origin of such a property variation between different interfaces. To answer this question it is necessary to get an insight into the intrinsic structure of interfaces. We can assume that the interfacial structure varies with misorientation and the observed cusps indicate that there exist the boundaries with exceptional (special) structures and properties.

The next section describes the commonly used models of interfacial structure that are supposed to deal with their properties.

\section{Structure of intercrystalline interfaces}

\subsection{Geometry of interfaces}

An interface between two crystals possesses in a general case 8 degrees of freedom (DOF) $[33,34]$. In order to characterise geometrically a grain boundary it is necessary to define the following parameters: misorientation axis (2 DOF), misorientation angle ( $1 \mathrm{DOF})$, the normal to grain boundary plane (2 DOF), magnitude and orientation of rigid body translation between two grains (3 DOF). Another description of the first $5 \mathrm{DOF}$ is based on the interface plane orientation with respect to each grain and the relative rotation between grains. It is especially convenient to describe the geometry of interphase boundaries. These descriptions allow us to determine whether the boundary is symmetrical or asymmetrical, or what their tilt and twist components are [33].

It must however be underlined that this geometrical description does not give any indication about the energy of the interface. Hence, great efforts were made to construct structural models that enable one to predict interfacial properties.

\subsection{Models of interfacial structure}

Among different structural models that have been proposed in the scientific literature only the most widely used models are described in this section. 


\subsubsection{Dislocation and disclination models}

A dislocation model is the first rigorous model of grain boundaries that enable one to quantify the GB's energy [35]. For a boundary composed of a set of parallel edge lattice.dislocations the energy $\gamma_{b}$ can be expressed by the following equation:

$\gamma_{b}=G b \theta(A-\ln \theta) / 4 \pi(1-\nu)$,

where $b$ is the magnitude of dislocation Burgers vector, $\theta$ is the misorientation angle, $G$ is the shear modulus, $\nu$ is the Poisson ratio and $A$ is a constant. This equation indicates that the energy increases with misorientation angle. The model was successfully applied to boundaries with misorientation angles lower than $15^{\circ}$. For greater misorientation angles when the dislocation cores overlap it does not give the correct values of GB energy. It can be noticed that on the basis of this model a new GB disinclination model has been developed, which gives a good description of the GB energy and GB stresses for high misorientation angles [36].

\subsubsection{Coincidence models}

To describe the structure of the large angle boundaries a coincidence site lattice (CSL) model is more useful $[23,26]$. This is the most widely used in the studies of relationships between GB crystallography and their properties. It is based on a three-dimensional set of coincidence sites, when two crystals are supposed to interpenetrate. The ratio of the coincidence unit cell volume to the crystal unit cell volume called coincidence index $\Sigma$ is a principal parameter in the model. In other words it is equal to the reciprocal density of coincidence lattice sites relative to crystal lattice sites. This means that for a low value of $\Sigma$ there is a high density of crystal lattice sites in coincidence (Fig. 2). Such boundaries are supposed to have low energy and are called "special" boundaries. The model is based on the assumption that the boundary energy increases monotonically with the coincidence index $\Sigma[37]$.

Another coincidence model was constructed on the criterion of coincidence of lattice planes which match across the boundary. This is the so-called "plane matching" (PM) model [38]. The boundaries of low energy are those, for which matching planes have low crystallographic indices and are perpendicular to the boundary plane. In such a case the normal to these planes, which is the coincidence axis direction, lie in the boundary plane.

It can be easily shown that even a small deviation from the exact coincidence misorientation should completely destroy the coincidence of sites. This effect should in turn abruptly rise boundary energy, which is not observed experimentally. Hence, the CSL model was later extended to GBs with misorientations near to coincidence relationship by introducing the hypothesis that the deviation from coincident misorientation is accommodated by a regular array of intrinsic grain boundary dislocations (IGBDs) [39]. These boundaries can be considered as coincidence boundaries with superimposed networks of IGBDs which provide for the deviation angle $\Delta \theta$ from exact coincident misorientation. Such arrays of dislocations were later observed experimentally (Fig. 3). The energy $\gamma_{b}$ of near-coincidence boundaries containing IGBDs is equal to a sum of the elastic energy of the dislocation network, the core energy of the dislocations and the energy 


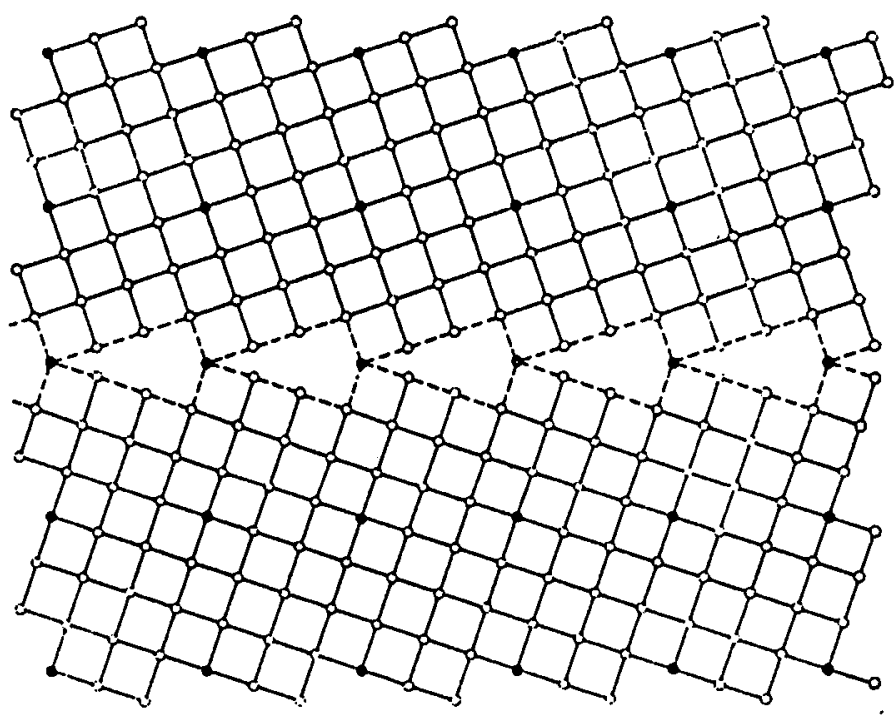

Fig. 2. Schematic representation of a $36.9^{\circ}\langle 100\rangle$ tilt boundary between two fcc crystals. The sites of the coincidence are indicated by black circles.

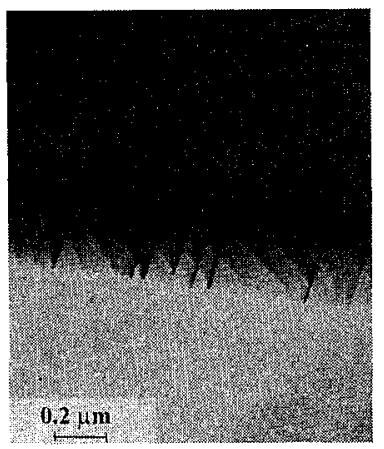

Fig. 3. A quasi periodic array of intrinsic GB dislocations and some irregularly spaced extrinsic dislocations in a $48.5^{\circ}\langle 110\rangle$ tilt boundary deviated by $2^{\circ}$ from $\Sigma 11$ coincident misorientation.

of the reference coincident boundary $\gamma_{b}^{0}$. In the case of tilt boundaries the energy is given by equation of the following form [40]:

$$
\gamma_{b}=\gamma_{b}^{0}+B b \Delta \theta(A-\ln \Delta \theta),
$$

where $b$ is the magnitude of the IGBD Burgers vector, $A$ and $B$ are parameters depending on material constants.

Equation 6 shows that energy increases with the deviation angle $\Delta \theta$. The boundaries with misorientations far from the coincidence relationship, for which the IGBD cores overlap, possess high energy and are classified as "general" GBs.

The concept of coincidence was also extended to non-cubic crystal structures, 
for which the exact coincidence does not exist. In this case the mathematically exact CSL boundaries can only be obtained by applying a homogeneous strain $\varepsilon$ to at least one of the crystals or by introducing the set of IGBDs at the interface plane [41]. The same approach was adopted for interphase boundaries, for which the interatomic spacings of one phase are incommensurate with those of the other in the interfacial plane. In this case two coincidence indexes must be specified in respect to each phase [42]. This approach is known as a constraint coincidence site lattice (CCSL) model.

A more general and rigorous approach has been proposed by Bollmann [43], who considers not only lattice sites, that are invariant during transformation from one crystal to the other, but all invariant points, called " $O$ " points, of such transformation. This model is therefore called the "O-lattice" theory. In this sense the coincidence site lattice is a sub-set of " $O$ "-lattice. The GB regions between " $O$ " points constitute the so-called "primary dislocations" and in this sense the theory is well connected with the dislocation model of GBs. The dislocations that accommodate the deviation from exact coincidence misorientation are called "secondary grain boundary dislocations" (SGBD). The great advantage of the theory is that it enables one to calculate the Burgers vector of secondary dislocations for any misorientation. It was shown that the Burgers vectors of intergranular dislocations belongs to the displacement shift complete (DSC) lattice, which represent all translation vectors between two crystal lattices. The main field of application of coincidence and "O"-lattice models are therefore the analysis of intergranular dislocations and the processes in which they participate, such as plastic deformation, GB sliding and migration.

Independently of the relative success, the geometrical models have received some criticism. First of all the strict relationship between the $\Sigma$ value and the GB energy was not demonstrated theoretically nor experimentally. Moreover the models do not give any insight into the atomic structure of the boundary. In fact, the computer simulations of $\mathrm{GB}$ atomic structures have demonstrated that the GB structure relaxes in order to minimise the energy of the system [44]. The relaxation may consist of rigid body translation that destroy the geometrical coincidence of sites even if it preserves the periodicity of a boundary.

\subsubsection{Structural unit model}

The insufficiencies of geometrical (CSL, PM and "O"-lattice) models have stimulated the development of the new model based on energetic considerations. It supposes that when two crystals are joined together, the atoms of two crystals interact at the interface and tend to take the new positions of minimum energy. The new atomic configurations created at the interface differ from these in the crystal lattice. They form the compact polyhedral units called "structural units" which are periodically repeated in the boundary. Computer simulations of the atomic structure of boundaries based on the minimization energy of the system, have confirmed this idea $[44,45]$.

There are some correlations between the structural unit (SU) and geometrical models, though they are constructed on different assumptions $[45,46]$. For example, a low $\Sigma$ coincident boundary corresponds to the intergranular structure 
composed with only one type of structural units (A). This special GB is called a "favoured" boundary. Units (A) are supposed to be the cores of primary dislocations. When the misorientation angle deviates from the exact coincidence, the new additional units (B) appear periodically in the boundary. These are the cores of secondary dislocations that accommodate the deviation from the special misorientation. The boundaries composed of two or more structural units have increased energy and are called "non-favoured" boundaries. It follows that in some cases these different approaches describe the same reality in different terms. The use of different models is however justified by the fact that they are more or less appropriate to describe different properties and processes occurring at interfaces. For example, the SU model is well adapted to study the boundary interactions with foreign atoms, whereas the "O"-lattice model is mostly used to analyse the intergranular dislocations [46].

It can be concluded that a realistic boundary structure can be found theoretically by means of computer simulations, when a physically reliable description of interatomic potentials is used. The SU model has been confirmed in the case of tilt, low periodic GBs by use of the high resolution electron microscopy (HREM). It provides a good approach for defining low energy boundaries [47]. The use of this model to classify general GBs (with high index misorientation and/or mixed tilt-twist character) is however not straightforward.

\subsection{Criteria of low interfacial energy}

The structure of interfaces changes with the geometrical parameters (misorientation, GB plane). According to the model used the changes consist on variation of the density of coincident sites, content and type of intergranular dislocations, kind of structural units and the atomic density of GB plane. All the models try to predict the interfaces of low energy. Thus, on the basis of the models described several geometrical criteria of low interfacial energy have been proposed in order to select interfaces with special properties [29].

In the frame of coincidence model, two criteria of low interfacial energy has been proposed: (1) low value of $\Sigma$ index which defines the inverse of the density of coincident lattice sites or (2) high specific density of coincidence sites in the boundary plane $\Gamma(h k l)=1 / A(h k l)$, where $A(h k l)$ is the boundary plane area associated with one coincident lattice site. The last criterion seems to correlate better with GB energy than $\Sigma$, because it corresponds to the density of atoms at shared sites and to the structural periodicity in the GB plane. Many experimental studies reveal deep cusps of grain boundary energy for some low $\Sigma$ boundaries. It was however been observed that not all coincident boundaries do display low energy. Inversely, there are some boundaries with high $\Sigma$ and relatively low energy $[24,29]$.

Alternatively, the low interfacial energy can be attributed to interfaces for which dense planes and directions of both grains (1) and (2) are parallel in the boundary [48]. This criterion called a "planar criterion" correlate better with the structural unit model $[46,49]$. The parameter indicating the low energy is the high value of mean interplanar spacing at the interface: $d_{\text {eff }}=d\left(h_{1} k_{1} l_{1}\right)+$ $d\left(h_{2} k_{2} l_{2}\right) / 2[34,50]$. This happens for boundaries that match dense, low index 
planes together. Such boundaries are composed of small structural units repeated with high periodicity.

In some cases different criteria may be equivalent. For example in $\langle 011\rangle$ and $\langle 001\rangle$ tilt grain boundaries, where there is one crystal lattice site for every coincident site in the boundary $\Gamma(h k l)=d(h k l) / \Omega$. This means that in this case the "planar coincidence site density, $\Gamma$ " criterion is equivalent to "interplanar spacing $d_{\text {eff }}$ " criterion. However, this is not always true as in the case of some asymmetrical boundaries. In fcc system for example, a grain boundary with the plane $\{111\}_{1} /\{001\}_{2}$, for which $\Gamma=0$, attains a relatively high value of $d_{\text {eff }}$. This means that the use of geometrical criteria is not straightforward and must be applied very carefully. Moreover, it must to be outlined that any geometrical models do not allow one to classify all kinds of interfaces according to their energy.

The applicability of a given criterion depends on the kind of material and the type of interface (GB or IB). It is possible to select a suitable criterion with help of a "localization parameter $p$ " proposed by Eojkowski [48]:

$$
p=E_{\mathrm{AB}} / \Omega G,
$$

where $E_{\mathrm{AB}}$ is the cohesive energy across the interface, $\Omega$ is the atomic volume and $G$ is the shear modulus of the crystal.

This concept based on physical background, explicitly accounts for interaction of the atoms across the interface and permit a selection of the better criterion for a given material. It was shown that for $p>0.3$ the low energy interfaces obey the coincidence $(\Sigma, \Gamma)$ criteria. For $p<0.3$ the planar criterion governs the energy of interfaces [48].

It follows that by producing the boundaries according to these geometrical criteria it would be possible to control the energy of interfaces and other related interfacial properties. The dependence on geometrical parameters has been observed for such intergranular properties as diffusion $[23,25]$, cohesion [9], corrosion $[4,11]$, sliding [30] and others.

\section{Interface microstructure}

The interfacial structure has a quasi-bidimensional (2D) character, which implies that their defects are essentially 0 -dimensional as vacancies or foreign atoms and linear (1D), i.e. dislocations. One can also classify as an element of the interface the $2 \mathrm{D}$ defects as steps and $3 \mathrm{D}$ as intergranular phases. A whole set of these defects constitutes the interfacial microstructure.

The structure of vacancies depends on the structure of the interface: in well ordered, low-energy interfaces vacancies have localised the structure but in high energy disordered boundaries they are relaxed [51], which means that they are spread over several atomic distances. The general boundaries are considered to be the perfect sources and sinks for vacancies. This effect may have some implications in such properties as intergranular diffusion and high temperature creep [52].

A strong influence on interfacial properties may have foreign atoms [53]. The structure of interfaces is generally more open than those of the crystal, and therefore can dissolve higher concentrations of foreign atoms compared to crystal lattice. The enrichment of the boundary in foreign atoms is called intergranular 


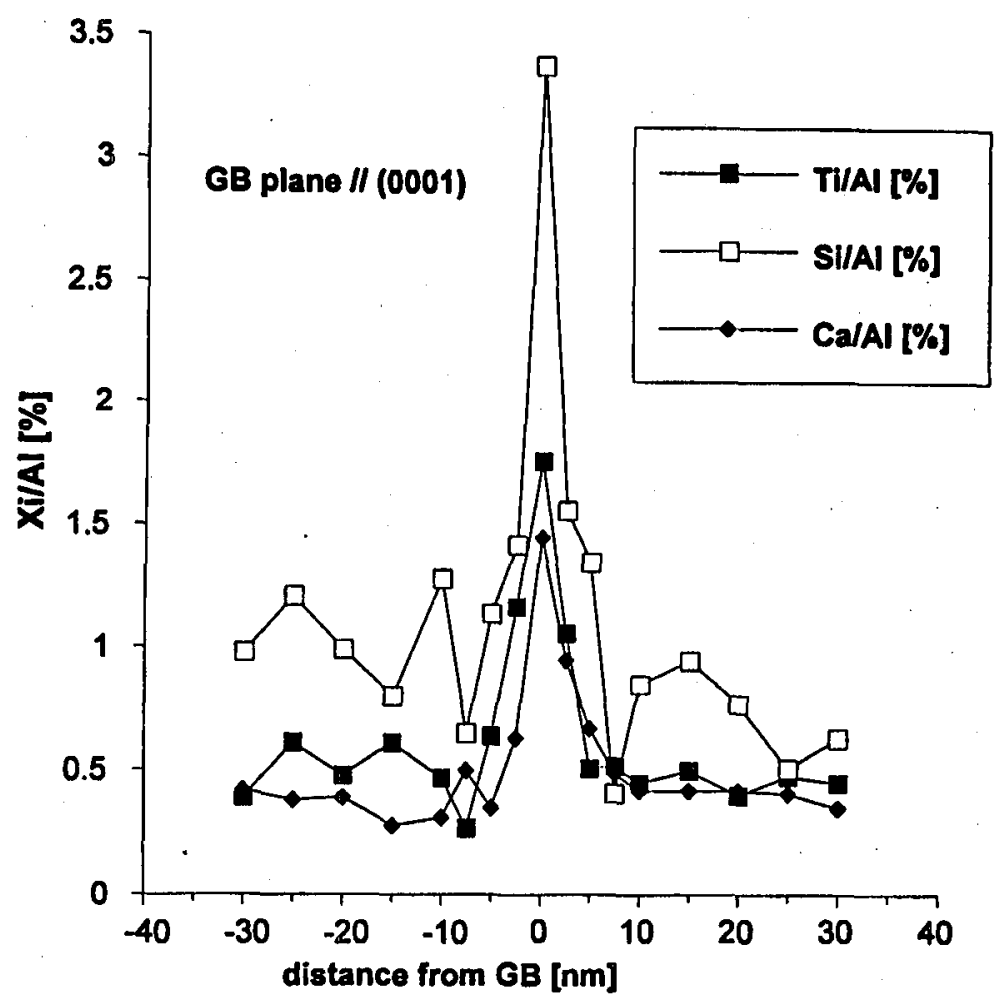

Fig. 4. Concentration profiles of $\mathrm{Ti}, \mathrm{Si}$ and $\mathrm{Ca}$ across a grain boundary in $\mathrm{Al}_{2} \mathrm{O}_{3}$ showing the GB segregation of these elements [53].

segregation. An example of intergranular segregation detected by an X-ray microanalysis is given in Fig. 4.

Interfacial segregation may drastically alter the interfacial properties by modification of atomic and electronic structure of the interface [54]. This may reduce the interfacial cohesion as in the case of sulphur segregation in nickel [55] or increase in it as in the case of boron segregation in $\mathrm{Ni}_{3} \mathrm{Al}[56,57]$. The segregation modifies also the electrochemical properties of interfaces and their corrosion resistance [58]. It is generally assumed that the segregation reduces interfacial energy, which in turn leads to the decrease in GB diffusivity and other kinetic properties such as a boundary migration [58].

It has been observed that the interfacial segregation occurs easier on general, high energy boundaries than on special, low energy boundaries [25]. It is worth noticing that not only does the segregation depend upon the intergranular structure, but inversely, the GB atomic structure may be strongly altered by segregation [59].

Intrinsic dislocations that accommodate the deviation from coincidence misorientation, described in Sec. 3.2.2, constitute the intrinsic element of GB structure. The characteristic feature of these intrinsic defects is that they form a regular, 
periodic array which results in the cancelling of long range stress fields of dislocations.

Interfaces may also contain extrinsic dislocations (Fig. 4), which do not form such a regular array in the interfacial plane, and therefore display long range stresses. Most of them originate from lattice dislocations which move during plastic deformation towards the interfaces, where they are trapped. The trapped lattice dislocation (TLD) tends to dissociate into intergranular dislocations with DSC Burgers vectors [12].

The mobility of intergranular dislocations controls many high temperature processes such as grain growth, intergranular sliding, superplasticity and dynamic recovery $[12,60]$. As the mobility of intergranular dislocations depends on interfacial diffusivity [15] it can also be controlled by the geometrical parameters related to low interfacial energy. Indeed, it has been well documented that the mobility of intergranular dislocations is reduced in low $\Sigma$ coincident boundaries and increased in general boundaries [12].

However, recent experimental studies have shown that the mobility of intergranular dislocations is stronger influenced by intergranular segregation than by crystallographic factors [61]. It means that in the presence of intergranular segregation the geometrical characteristics of GBs do not permit us to predict its properties. In other words the purely geometrical criteria of GB speciality fail in the presence of intergranular segregation [61].

The interfacial ledges with a height of several interatomic distances can be considered as the bidimensional elements of interfacial microstructure. Generally the interfacial steps are involved during GBs migration [23, 26]. In the case of interphase boundaries the ledges play an important role in the phase transformations.

Interfaces may also display intergranular phases in form of precipitates or continuous intergranular layers. These are known to strongly influence the material properties. For instance, the intergranular phases in some ceramics may constitute the favourable path for leakage currents deteriorating their insulating properties [62]. On the contrary, in polycrystalline ceramic superconductors GBs constitute strong resistive barriers and reduce the critical current densities $J_{c}[7,8]$, which limits the application of these materials. It should be pointed out that the formation of second intergranular phases may sometimes be beneficial, as in the case of hyperfrequency properties of ferrites [18].

It has been observed that the nucleation and growth of these phases depend strongly on the structure of boundaries (grain's misorientation or GB plane orientation) at which they are formed [26]. For high energy boundaries, the interfacial energy minimization can be reached via the formation of intergranular phases according to the criterion: $\gamma_{b}>2 \gamma_{i}$, where $\gamma_{i}$ is the energy of crystal/intergranular layer interface [62]. On the contrary for low energy coincident boundaries the kinetics of second phase's formation may be strongly reduced.

\section{Summary and concluding remarks}

The interface engineering is based on structural effects on interface properties and adopts the following philosophy; the crystallographic structure of an interface defined by grains' misorientation and interface's plane orientation engen- 
ders specific properties. These are different from the properties of neighbouring grains and from properties of other interfaces with different crystallography.

The structure of interfaces changes as a function of their crystallography. The changes consist of variation of the coincident site's density, the type and content of intergranular dislocations or the kind of structural units forming the interface. This enables one to control the interfacial energy and other related properties using structural criteria of low interfacial energy.

Furthermore, each interface exhibits characteristic defects, which are different from those of the crystal lattice. The nature, structure and density of interfacial defects vary as a function of interface crystallography. The whole set of interfacial defects is called an interface microstructure.

The interfacial properties result from the combined effects of interface crystallography and interfacial microstructure. These factors are mutually related, i.e. crystallography of interfaces influences the interfacial microstructure and vice versa. The knowledge of interfacial crystallography is necessary but insufficient to predict interfacial properties. The interfacial microstructure may influence the properties stronger than the crystallographic structure of interfaces.

The influence of interfacial properties on the macroscopic behaviour of a material is exercised in a general case via complex processes involving mutual interactions between interfaces, theirs defects and other elements of the material microstructure. To efficiently control the influence of interfaces on material properties it is necessary to perform a careful analysis of these processes at microscopic level in order to appropriately select these structural factors of interfaces that govern these processes and the macroscopic response of the material.

\section{Acknowledgments}

The author is indebted to Prof. M.W. Grabski for inspiration for the subject and for stimulating and fruitful discussions. The author would also like to thank Prof. L. Priesfer and Dr J.Y. Laval for many fruitful conversations. This work was partly supported by the Committee for Scientific Research, grant No. 3P40704005.

\section{References}

[1] M.W. Grabski, Istota Inżynierii Materiatowej, Oficyna Wydawnicza Politechniki Warszawskiej, Warszawa 1995 (in Polish).

[2] T. Watanabe, Res Mech. 11, 47 (1984).

[3] M.W. Grabski, J. Phys. (France) 46, C4-567 (1985).

[4] G. Palumbo, K.T. Aust, Acta. Metall. Mater. 38, 2343 (1990).

[5] L.K. Fionova, A.V. Artemyev, Grain Boundaries in Metals and Semiconductors, Les Editions de Physique, Les Ulis 1994.

[6] D. Dimos, P. Chaudhari, J. Mannhart, F.K. LeGoues, Phys. Rev. Lett. 61, 219 (1988).

[7] J.Y. Laval, M. Drouet, W.A. Świątnicki, J. Phys. (France) III 4, 2195 (1994).

[8] J.Y. Laval, M. Drouet, W.A. Świątnicki, C. Cabanel, Physica C 235-240, 2987 (1994).

[9] T. Watanabe, Mater, Sci. Eng. A 176, 39 (1995). 
[10] N.L. Peterson, in: Grain Boundary Structure and Kinetics, Ed. R.W. Balluff, ASM Metals Park, Ohio 1980, p. 209.

[11] M. Yamashita, T. Mimaki, S. Hashimoto, S. Miura, Philos. Mag. A 63, 695 (1991).

[12] M.W. Grabski, J. Phys. (France) 49, C5-497 (1988).

[13] W.A. Świątnicki, M. Styczyńska, M.W. Grabski, Acta Metall. 33, 1643 (1985).

[14] W.A. Świątnicki, Ph.D. Thesis, Warsaw University of Technology, Warsaw 1986.

[15] W.A. Świątnicki, W. Łojkowski, M.W. Grabski, Acta Metall. 34, 599 (1986).

[16] K.J. Kurzydłowski, R.A. Varin, W. Zieliniski, Acta Metall. 32, 71 (1984).

[17] C. Cabanel, J.Y. Laval, J. Appl. Phys. 67, 1425 (1990).

[18] M.H. Berger, J.Y. Laval, J. Phys. (France) 51, C1-965 (1990).

[19] P.J. Goodhew, T.Y. Tan, R.W. Balluffi, Acta Metall. 26, 1113 (1978).

[20] P.J. Goodhew, Metal Science 13, 108 (1979).

[21] M. Sautter, H. Gleiter, G. Baro, Acta Metall. 25, 467 (1977).

[22] W. Łojkowski, H. Gleiter, J. Phys. (France) 46, C4-107 (1985).

[23] H. Gleiter, B. Chalmers, in: High Angle Grain Boundaries, Eds. B. Chalmers, J.W. Christian, T.B. Massalski, in series Prog. Mater. Sci., Vol. 16, Pergamon Press, Oxford 1972.

[24] P.J. Goodhew, in: Grain Boundary Structure and Kinetics, Ed. R.W. Balluff, ASM Metals Park, Ohio 1980, p. 155.

[25] G. Palumbo, K.T. Aust, in: Materials Interfaces. Atomic-Level Structure and Properties, Ed. D. Wolf, Chapman \& Hall, London 1992, Ch. 5.

[26] L.E. Murr, Interfacial Phenomena in Metals and Alloys, Addison-Wesley Publishing Company, London 1975, Ch. 5.

[27] D. Gupta, Philos. Mag. 33, 189 (1976).

[28] G. Martin, B. Perraillon, in: Grain Boundary Structure and Kinetics, Ed. R.W. Balluffi, ASM Metals Park, Ohio 1980, p. 239.

[29] A.P. Sutton, R.W. Balluffi, Acta Metall. 35, 2177 (1987).

[30] P. Lagarde, M. Biscondi, J. Phys. (France) 36, C4-297 (1975).

[31] G. Dhalenne, M. Déchamps, A. Revcolevschi, in: Grain Boundaries in Semiconductors, Eds. H.J. Leanny, G.E. Pike, C.H. Seager, North-Holland, New York 1982.

[32] A. Roy, U. Erb, H. Gleiter, Acta. Metall. 30, 1850 (1982).

[33] F.F. Lange, Acta Metall. 15, 311 (1967).

[34] D. Wolf, in: Materials Interfaces. Atomic-Level Structure and Properties, Ed. D. Wolf, Chapman \& Hall, London 1992, Ch. 1.

[35] W.T. Read, W. Shockley, Phys. Rev. 78, 275 (1950).

[36] J.A. Hurtado, B.R. Elliot, H.M. Shojda, D.V. Gorelikov, C.E. Campbell, H.E. Lippard, T.C. Isabell, J. Weertmann, Mater. Sci. Eng. A 190, 1 (1995).

[37] A. Brokman, R.W. Balluffi, Acta Metall. 29, 1703 (1981).

[38] P.H. Pumphrey, in: Grain Boundary Structure and Properties, Eds. G.A.Chadwick, D.A. Smith, Academic Press, New York 1976, p. 139.

[39] D.G. Brandon, Acta Metall. 14, 1479 (1966).

[40] R.W. Balluff, T.Y. Tan, Scr. Metall. 6, 1033 (1972). 
[41] F.R. Chen, A.H. King, Philos. Mag. A 57, 431 (1988).

[42] R.W. Balluff, A. Brokman, A.H. King, Acta Metall. 30, 1453 (1982).

[43] W. Bollmann, Crystal Defects and Crystalline Interfaces, Springer-Verlag, Berlin 1970.

[44] R.C. Pond, V. Vitek, Proc. R. Soc. Lond. B 357, 453 (1977).

[45] A.P. Sutton, Int. Met. Rev. 29, 377 (1984).

[46] L. Priester, Rev. Phys. Appl. 24, 419 (1989).

[47] A.P. Sutton, V. Vitek, Philos. Trans. R. Soc. Lond. A 309, 1 (1983).

[48] W. Lojkowski, Struktura granic między kryształami, Centrum Badań Wysokociśnieniowych PAN, Warszawa 1992 (in Polish).

[49] V. Paidar, Philos. Mag. A 66, 41 (1992).

[50] V. Paidar, Phys. Status Solidi A 92, 115 (1985).

[51] H. Gleiter, in: Physical Metallurgy, Eds. R.W. Cahn, P. Haasen, North-Holland Physics Pub., Amsterdam 1983, Ch. 10B.

[52] E. Arzt, M.F. Ashby, R.A. Verall, Acta Metall. 31, 1977 (1983).

[53] W.A. Świątnicki, S. Lartigue-Korinek, J.Y. Laval, Acta Melall. Mater. 43, 795 (1995).

[54] C.L. Briant, in: Materials Interfaces. Atomic-Level Structure and Properties, Ed. D. Wolf, Chapman \& Hall, London 1992, Ch. 17.

[55] T.C. Lee, I.M. Robertson, H.K. Birnbaum, Acta Metall. 37, 407 (1989).

[56] C.T. Liu, C.L. White, J.A. Horton, Acta Metall. 33, 213 (1985).

[57] W.A. Świątnicki, M.W. Grabski, Acta Metall. 37, 1307 (1989).

[58] D.F. Stein, L.A.Heldt, in: Interfacial Segregation, Ed. W.C. Johnson, ASM Metals Park, Ohio 1978, p. 239.

[59] W.A. Świątnicki, S. Lartigue, M. Biscondi, D. Bouchet, J. Phys. (France) 51, C1-341 (1990).

[60] W.A. Świątnicki, M.W. Grabski, Mater. Sci. Eng. 100, 85 and 93 (1988).

[61] W.A. Świątnicki, L. Priester, M.W. Grabski, in: Proc. 7th Intern. Conf. on Intergranular and Interphase Boundaries in Materials, IIB95, Lisbona, June 1995, to be published by Trans. Tech. Publ.

[62] J.Y. Laval, in: Charactérisation des poudres et des céramiques, Ed. J.L. Chermant, Hermes, Paris 1992, Ch. 6. 